\title{
Ciudad mancomunada Manta-Jaramijó-Montecristi Puerto HUB Mundial. Sostenibilidad y resiliencia de mayor densidad y compacidad urbana.
}

\author{
Alejandro Miguel Camino Solórzano ${ }^{1}$, Tatiana Cedeño Delgado ${ }^{2}$ \\ ${ }^{1}$ Rector. ${ }^{2}$ Facultad de Arquitectura. \\ Universidad Laica Eloy Alfaro de Manabi. Manta, Ecuador \\ E-mail: ${ }^{1}$ alejandro.camino@uleam.edu.ec, ${ }^{2}$ tatiana.cedeno@uleam.edu.ec
}

\begin{abstract}
Resumen. El puerto de Manta está situado en la punta más saliente de Ecuador y de América del Sur, cerca de la latitud 0, importante laboratorio urbano de las CIMEs Ciudades medias e intermedias. A este enclave geoestratégico oceánico se debe un desarrollo endógeno de ciudad puerto; de esplendor precolombino, secundario en la Colonia y en la República, explosivo en la modernidad a partir de su muelle de aguas profundas (1965) y sostenido desbordado en la contemporaneidad, pese a los esfuerzos de ordenamiento urbano a partir de los 90 (desde 1996 con apoyo de la Facultad de Arquitectura de la ULEAM) $y$ en medio de crisis políticas, geopoliticas, sociales y económicas del país. El crecimiento de la ciudad de Manta ha desbordado sus límites politicos hacia Jaramijó y Montecristi, crecimiento difuso y disperso; la propuesta planteada se constituye en una ciudad Mancomunada con una la estrategia BAAD Baja Altura Alta Densidad que permita ir hacia la compacidad.
\end{abstract}

Palabras clave: ciudad mancomunada, puerto $H U B$, planificación urbana y territorial CIMES, mayor densidad/ compacidad, BAAD baja altura alta densidad, habitabilidad.

\section{Introducción}

La ciudad de Manta en sus inicios fue un pequeño poblado de pescadores, dependiente administrativamente de Montecristi. En 1922 se separa de esta ciudad formando un nuevo cantón. A partir de ese momento Manta no ha dejado de crecer, ese proceso de desarrollo y expansión está en relación con el aprovechamiento de sus condiciones naturales y la construcción de infraestructuras que han generado finalmente crecimiento económico, atracción de inversiones foráneas y de población procedente de otros lugares del país y del mundo.

Manta es una ciudad de tamaño intermedio, que constituye el centro de un amplio territorio con el que se relaciona. Su desarrollo ha sido en algunos casos el resultado de la planificación y en otros la acción espontánea de diversos agentes, que han ido ocupando el territorio. Es decir, una parte ha sido planificada según diversas fórmulas (continuidad de la trama regular, incorporación de piezas completas destinadas al servicio de la ciudad, creación de infraestructuras viarias de conexión), la otra parte es el resultado de la adaptación al terreno y la voluntad de múltiples agentes, que han parcelado y vendido por trozos la tierra para edificar. Finalmente el resultado es la suma de numerosas piezas que en la actualidad interactúan entre sí y que forman un mosaico articulado por las infraestructuras y la vialidad actual de la ciudad.

El consumo de suelo en la ciudad desde el año 1974 al 2018 fue de 4903 hectáreas, la densidad descendió casi 9 puntos y la población se incrementó $370 \%$. La expansión urbana de la ciudad traspasa los límites políticos administrativos con lo cual se forma 


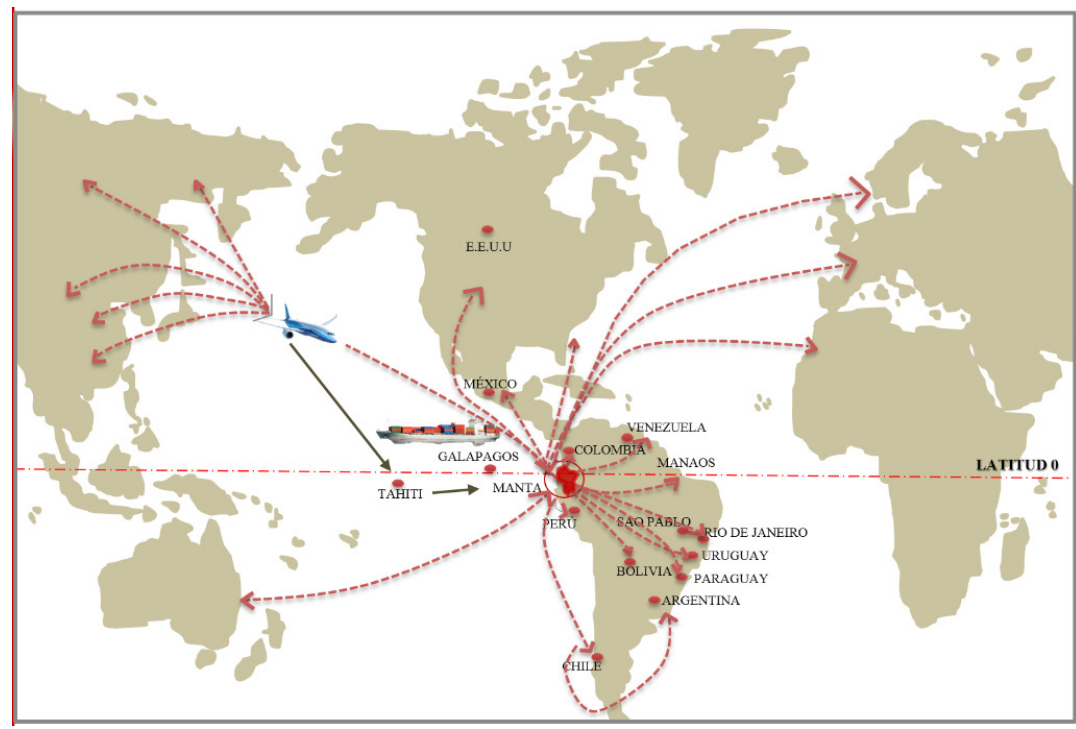

Fig. 1. Ubicación estratégica de la ciudad de Manta en el mundo. Fuente: Plan Estratégico Manta 2020.

la conurbación Manta - Montecristi - Jaramijó.

De acuerdo a las proyecciones poblacionales del INEC y Yanez, la población de la ciudad mancomunada (Manta, Montecristi, Jaramijó) en el 2020 es de 412157 habitantes que se encuentran asentados en una superficie de 9875,38 ha. lo que constituye una densidad de $41 \mathrm{hab} / \mathrm{ha}$. Asimismo, la población proyectada al año 2050 incrementa casi el $300 \%$ de la población existente en el año 2010 , lo que refleja que se deben tomar estrategias para desarrollar una ciudad mancomunada compacta, sostenible que permita albergar

\section{Conceptualización}

Densidad.- Número de individuos de la misma especie que viven por unidad de superficie.
(RAE, 2019). Los tres indicadores más comunes en el urbanismo son: la densidad poblacional (número de habitantes por unidad de superficie), densidad residencial o de vivienda (número de viviendas por unidad de superficie) y la densidad o intensidad edificatoria, también conocida como edificabilidad (superficie construida por unidad de superficie) en donde la unidad de superficie que se suele utilizar es la hectárea.

Compacidad.- Expresa la idea de proximidad de los componentes que configuran la ciudad, es decir, de reunión en un espacio más o menos limitado de los usos y las funciones urbanas. (Agencia de Ecología Urbana, Barcelona)

Diversidad.- Se relaciona con la mixticidad, con la heterogeneidad. En ese sentido, habría que hablar de la necesidad de mezcla de usos

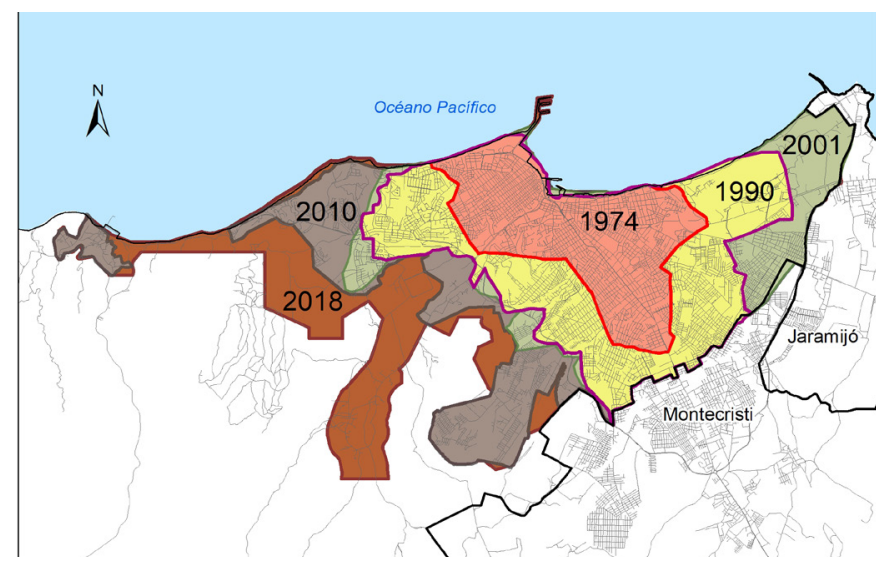

Fig. 2. Crecimiento de la ciudad de Manta desde el año 1974 al 2018. Fuente: INEC 1974, 1990, 2001, 2010, 2018. Elaboración: Autores
Tabla 1. Población, superficie y densidad de Manta desde el año 1974 al 2018 Fuente: INEC 1974, 1990, 2001, 2010. GAD Manta 2018. Elaboración: Autores

\begin{tabular}{|c|c|c|c|}
\hline Año & Población hab. & Superficie ha. & Densidad hab/ha. \\
\hline 1974 & 70015 & 1375,37 & 50,91 \\
\hline 1990 & 132816 & 3137,57 & 42,33 \\
\hline 2001 & 120322 & 3895,1 & 49,38 \\
\hline 2010 & 226477 & 5010,22 & 45,20 \\
\hline 2018 & 259052 & 6278,38 & 41,26 \\
\hline
\end{tabular}


Gráfico 1. Población de Manta, Montecristi, Jaramijó años 1990 - 2050. Fuente: INEC 1974, 1990, 2001, 2010. Yáñez 2019. Elaboración: Autores

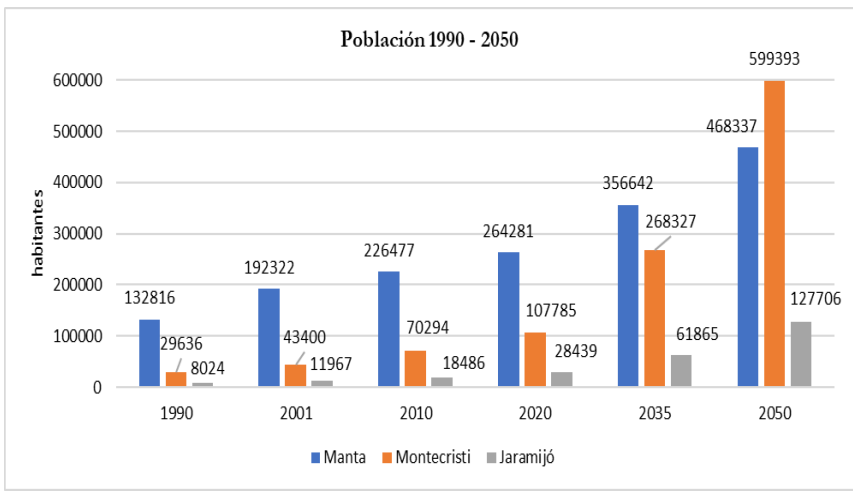

Gráfico 2 Población ciudad mancomunada 1990 al 2050. Fuente: INEC 1974, 1990, 2001, 2010, 2020. Yánez, 2019. Elaboración: Autores

y actividades (incluir usos terciarios repartidos por doquier en los barrios residenciales); mezcla de tipos de viviendas (para diferentes tipos de renta, para diferentes tipos de hogares, etc.); mezcla de personas (jóvenes, niños, ancianos, personas de mediana edad), etc.

BAAD Baja Altura Alta Densidad.- El concepto comienza a tener protagonismo a partir de mediados de los años 40, en lugar de conseguir una densidad residencial media o media-alta construyendo tipologías de vivienda colectiva en bloque, manzana o torre, se logran densidades similares con viviendas unifamiliares de una o dos plantas, sin renunciar a ello la calidad urbana de las intervenciones. (Rosario Del Caz, 2013)

Ciudad Mancomunada.- Ciudad que por efectos de crecimiento, desarrollo y expansión hacia sus límites y fronteras forma conurbación con las ciudades vecinas, y que por su estrecha interrelación y complementariedad social, económica, logística con ellas se

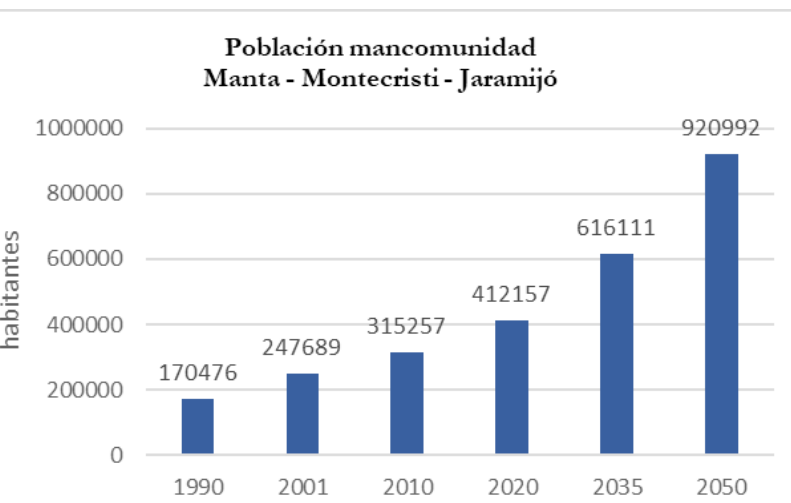

complementan formando mancomunidad para lograr un desarrollo integral, permitiendo reducir el desequilibrio territorial.

\section{Justificación}

Una característica de la expansión urbana es la baja densidad con la que se urbaniza, existe evidencia de que a nivel global, a pesar de los rápidos procesos de urbanización, el crecimiento urbano que se está dando en las ciudades cada vez es menos compacto, algo que se puede interpretar como desarrollo poco sustentable (UN-HABITAT, 2012, P. 6).

La ciudades manabitas no se alejan de esta realidad, se evidencia un crecimiento desordenado no planificado, en el caso de Manta los asentamientos humanos fragmentados $\mathrm{y}$ dispersos con respecto al centro, desarrollándose programas habitacionales cerrados generando la periferia dormitorio (la no ciudad). No obstante, con 
la dotación de redes de infraestructura (vías, agua potable, alcantarillado, energía eléctrica, transporte) constituye un factor económico considerable para los Gobiernos Autónomos Descentralizados e instituciones pertinentes a satisfacer.

La situación financiera del país no permite que los 221 GAD Cantonales puedan acceder a recursos económicos para implementar equipamientos como camales, mercados, plantas de agua, relleno sanitario, etc. Por ello, es prioritario que las municipalidades vecinas o circundantes se agrupen en mancomunidades de manera que se racionalicen los recursos y exista una mejor distribución de los mismos. Asimismo, plantear propuestas que frenen la expansión urbana, el crecimiento desordenado, la periferia dormitorio promoviendo ciudades más densas, compactas, seguras y sostenibles.

\section{La Planificación Urbana en Manta.}

En el Plan Estratégico Económico y Social Manta (1997), su estrategia principal era la de configurar a Manta como un importante centro direccional de la microrregión manabita y de la macro región centro y Sudamérica en continua mejora de la calidad de vida y del progreso de los ciudadanos; además de convertir a Manta en un Puerto de transferencia Internacional; la potenciación de su sector industrial y de servicios, destacando de manera enfática el potencial turístico natural de la ciudad de Manta.

En el año 2000 Manta ingresa a la Red de Ciudades Medias e Intermedias CIMES, como producto de aquello en el año 2014 en la ULEAM se dicta un Taller de capacitación en Planificación territorial y urbana con el aval de Senplades y la Cátedra Unesco de la Universidad de Lleida dirigido por Josep María Llop, con énfasis en la aplicación de la metodología "Plan Base".

En el año 2006, se lleva a efecto el Taller Internacional de Planificación de Extensión Urbana organizado por PNUD, BID, Miduvi, 7 Municipios Nacionales, en el cual con asesoría francesa se bosqueja en un mapa la expansión de la ciudad de Manta. (Fig. 3)

A partir del 2011 la Facultad de Arquitectura de la ULEAM tiene una fuerte interacción con la escuela de arquitectura y urbanismo de la Universidad de Valladolid a partir del postdoctorado del Arq. Miguel Camino; pasantías y movilidad de docentes, artículos $\mathrm{y}$ ponencias indexadas, y un proyecto de investigación financiado por la Unión Europea que permitió la dotación de un laboratorio de ensayo de materiales, elaboración y la publicación del libro "Hábitat Social, digno, sostenible y seguro en Manta, Manabí, Ecuador" (Sáenz\&Camino, 2013) en el cual Rosario Del Caz, efectúa un análisis histórico cultural del desarrollo de la ciudad de Manta y plantea la propuesta BAAD (Baja Altura Alta Densidad) de mantener baja altura edificatoria pero incrementando la densidad de lotes y manzanas; para lo cual establece los siguientes rangos:

Densidad baja $=0$ a 30 viv. $/$ ha $\times 5.7 \mathrm{miem} /$ fam $=0$ a 171 hab./ha

Densidad media $=31$ a 60 viv. $/$ ha $\times 5.7 \mathrm{miem} /$
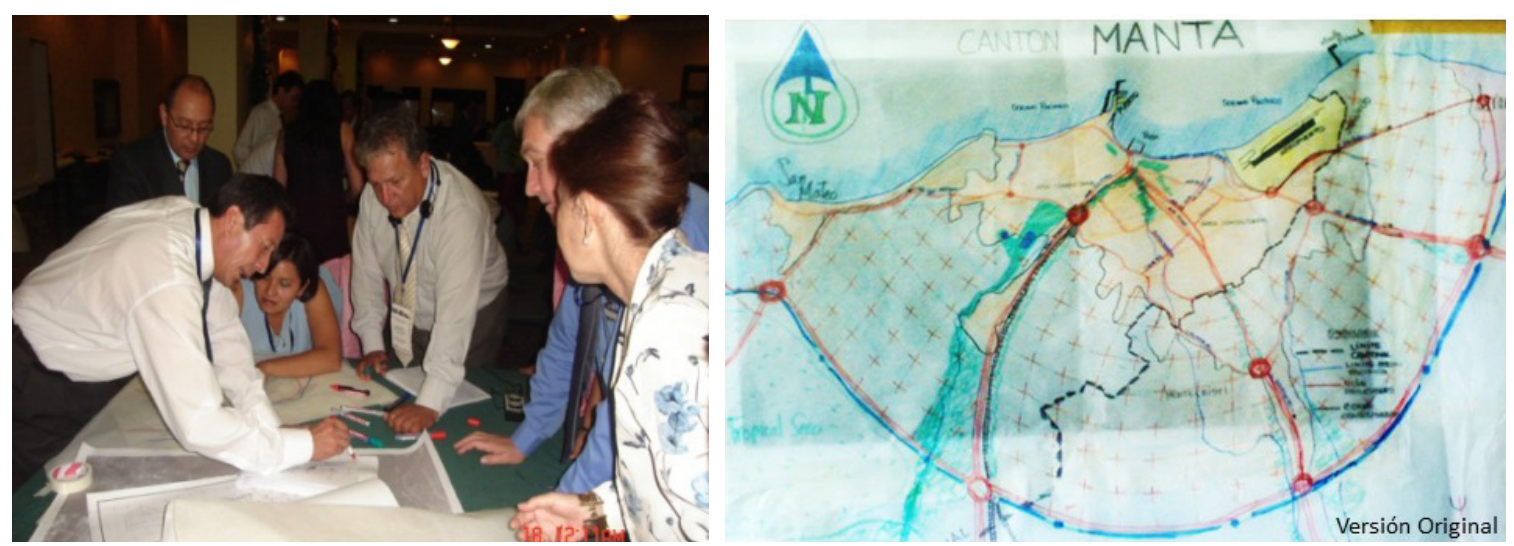

Fig. 3. Taller Internacional de Planificación Extensión Urbana (2006). Fuente: Camino Miguel, 2006. 
fam $=172$ a 342 hab./ha. Densidad alta $=61$ a $90 \mathrm{viv} . / \mathrm{ha} \times 5.7 \mathrm{miem} / \mathrm{fam}=342$ a $513 \mathrm{hab} . / \mathrm{ha}$.

Ventajas del Desarrollo BAAD, Ciudad Mancomunada para Manta.

- La no demanda de materiales o técnicas sofisticadas, así como de mano de obra especialmente cualificada.

-La agrupación de viviendas de baja altura proporciona una escala más humana, tanto del espacio privado como público.

- Posibilidad de implantación de sistemas de transporte público viables.

-Fomento de la vitalidad del barrio.

- Fomento del sentido de comunidad y mejora de la cohesión social.

-Reducción del consumo de suelo.

El Plan de Desarrollo Prospectivo de la ciudad de Manta 2019-2037-2050 recogió toda la planificación generada desde 1997; desde el modelo Barcelonés, puerto logístico internacional, ciudad moderna, equitativa e incluyente con un frente marítimo recuperado para el sector terciario de la economía, hasta la innovación y conectividad; tecnológica, humana, ambiental, emprendedora y participativa de Medellín; que se resume en el modelo territorial de sub-centralidades urbanas complementarias denominado "Collar de Perlas" que transforma la congestión del centro comercial formal e informal hacia actividades terciarias y turísticas relacionadas con este inmejorable enclave portuario, y su conexión con las sub-centralidades periféricas por medio de tres líneas de transporte público masivo digno.
El 16 de abril de 2016 la provincia de Manabí y Esmeraldas sufren el impacto de un terremoto de enorme magnitud (Pedernales $7.8^{\circ}$ en escala de Richter). La ciudad de Manta queda devastada tanto en servicios como en infraestructuras: hospitalarias, educativas, turísticas, comerciales, habitacionales, siendo demolidas edificaciones en el centro de Tarqui y en muchos barrios de la ciudad, perdiendo su memoria morfológica en la zona cero-Tarqui, no solo por la destrucción de sus edificios, sino porque también desapareció la vitalidad de su comercio formal e informal (3.000 comerciantes de la calle aproximadamente) que se ha trasladado a los barrios, demostrando que las propuestas desde 1997 y ahora reafirmadas en el "Collar de Perlas" son pertinentes, revitalizan la ciudad generando adicionalmente una seguridad pasiva.

\section{Propuesta "Ciudad Mancomunada Puerto HUB Mundial"}

Estrategias para el Desarrollo Urbano Ciudad Mancomunada Manta-Montecristi - Jaramijó.

1) Ocupación de terrenos baldíos en el centro de las 3 ciudades. (Ordenanza de ocupación inmediata) mayor densidad mayor compacidad (no menor a 5 pisos)

2) Incremento de altura edificatoria en el frente marítimo y a los lados de las principales arterias viales de la ciudad mancomunada.

3) Ocupación de la "Poza de Manta" bajo COS y alto CUS para el Down Town de Manta. (20 hectáreas)
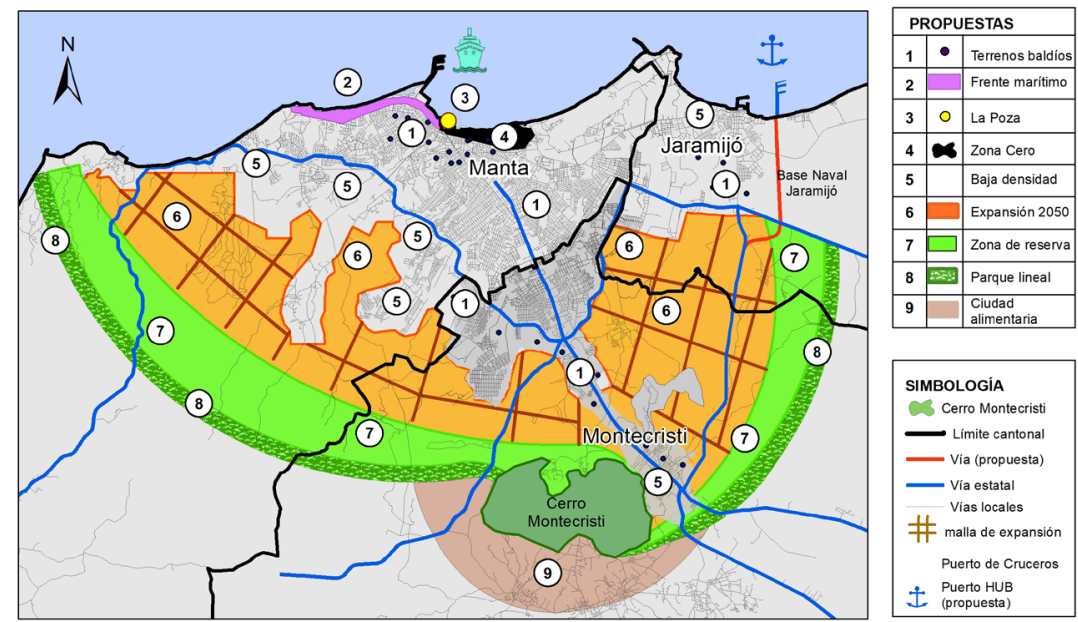

Fig. 4. Estrategias para el desarrollo urbano de la ciudad Mancomunada. Elaboración: Autores 


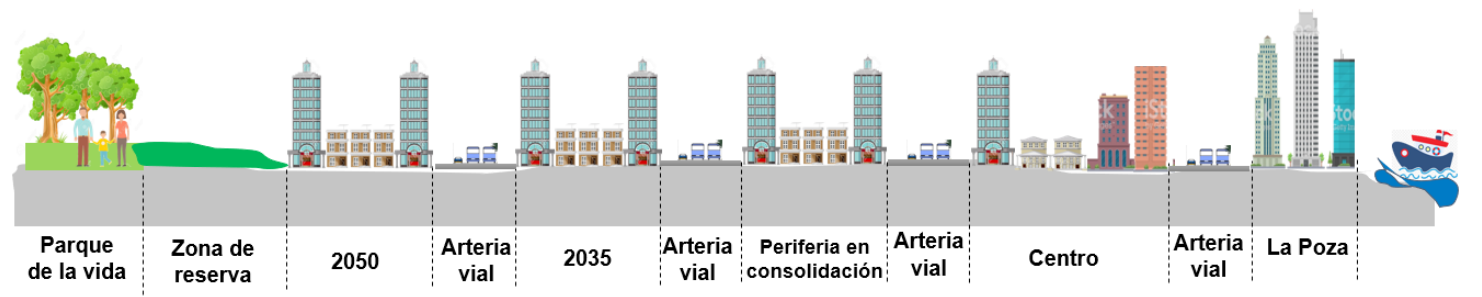

Fig. 5. Corte esquemático desarrollo ciudad mancomunada por zonificación y crecimiento de mayor densidad y compacidad. Elaboración: Autores.

4) Ocupación de la "Zona Cero de Tarqui" para uso terciario y vivienda; con énfasis en servicios turísticos y hoteles, espacios públicos, lúdicos y recreativos. (Mayor COS y CUS de 3 a 5 pisos de altura)

5) Incremento de uso y ocupación de suelo en la ciudad consolidada al 2019 al doble de densidad. (Manta, Montecristi, Jaramijó)

6) Expansión del suelo urbano al 2050 (antes suelo de reserva) con criterios BAAD.

7) Suelo de reserva, pulmón de la ciudad mancomunada.

8) Suelo frontera de reserva: parque lineal límite de la ciudad, de uso público masivo (500 m. de ancho) similar al Camino de la vida en Medellín.

9) Ciudad alimentaria con grupo visión Almería.

Con las estrategias planteadas se puede obtener como resultado que con el incremento de la altura edificatoria al doble (Municipios modifiquen el COS y el CUS) y la consolidación del área urbana de las 3 ciudades se puede duplicar la población actual a una cantidad de 700.000 habitantes aproximadamente, cifra mayor a la proyección poblacional estimada al 2035 de 616.111 habitantes (Yánez, 2019) esto sin necesidad de continuar expandiendo la mancha y polígono urbano de las ciudades (9875,38 hectáreas aproximadamente)

Se plantea un área de expansión urbana al año 2050 de 8413 hectáreas que solamente proyectando una densidad baja de $60 \mathrm{hab} /$ ha podría albergar a 500.000 personas más, constituyendo una ciudad mancomunada con un total de 1'200.000 habitantes (superior a los 920992 habitantes, cifra calculada por Yánez, 2019).
Infraestructuras de impacto y dinámicas de desarrollo y ordenamiento.

Para que la ciudad sea compacta debe expresar proximidad de los componentes que la configuran con los espacios para las distintas funciones urbanas. Se definen los siguiente roles para el desarrollo y consolidación de la ciudad Mancomunada.

1)Muelle de pesca y turístico de cruceros (existente)

2)Down Town "La Poza" Infraestructuras administrativas, culturales empresariales y lúdicas de gran escala que adolece la ciudad.

3)Zona Cero de Tarqui y malecón. Servicios Terciarios, Turísticos y Habitacional, devastada por el terremoto, sin reactivación hasta el momento.

4)Aeropuerto intercontinental y nacional en proceso de concesión con empresa coreana KAC.

5)Centro Técnico Administrativo Territorial de la Ciudad Mancomunada (con infraestructuras y equipamientos de la gran ciudad) en terrenos donados por los municipios en el punto geográfico donde convergen las tres ciudades.

6)Puerto Pesquero Artesanal "Jaramijó". (Existente) Que se debe potenciar.

7)Nuevo Muelle Puerto HUB de la Ciudad Mancomunada (Puerto Seco conectado a ZEDE) en terreno adjunto al nuevo campus ULEAM de Jaramijó

8)Campus ULEAM del Mar e institutos técnicos y de investigación oceánica del Pacífico Sur (128,05 ha)

9)ZEDE Zona Especial de Desarrollo Estratégico "Puerto HUB" adjunto al nuevo muelle de Aguas Profundas. 


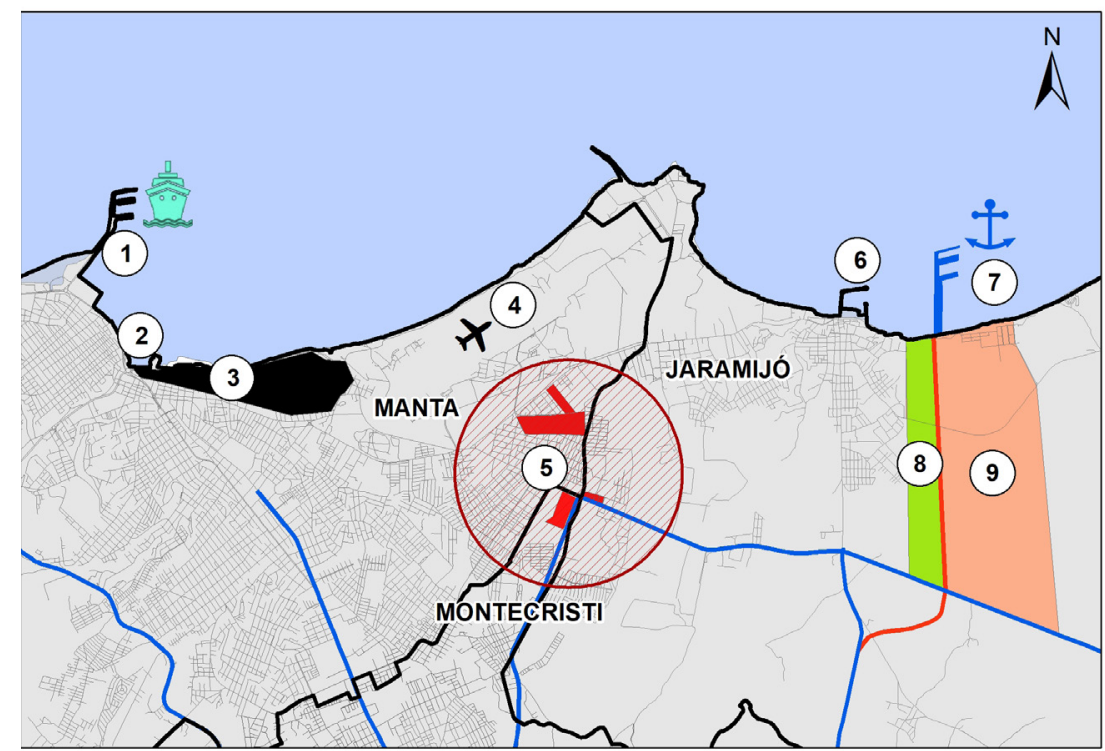

Fig. 6. Infraestructura logística Ciudad Mancomunada. Elaboración: Autores.

\section{Conclusiones y recomendaciones}

La ciudad de Manta no puede continuar expandiendo su malla urbana, creciendo desordenadamente, con asentamientos humanos dispersos, con déficit en infraestructuras y servicios básicos, invadiendo territorios rurales, recursos ambientales, agrícolas $\mathrm{y}$ acuícolas, causando impactos sociales y ambientales y de vulnerabilidad, que implican grandes e innecesarias inversiones económicas de mitigación a la ciudad.

El modelo propuesto es posible con la participación y compromiso de los GAD Municipales, instituciones, empresas públicas y privadas, universidades, cámaras de la producción, sector inmobiliario y comunidad; que desde cada sector promuevan y contribuyan a la nueva ciudad mancomunada compacta, segura, sostenible y resiliente.

El Estado debe contribuir con los proyectos e infraestructuras logísticas de la ciudad mancomunada y su área de influencia e intermediación, con un visión de desarrollo del país, a través de la complementariedad con las centralidades ya consolidadas (Quito, Guayaquil, Cuenca) potenciando la nueva centralidad Latitud Cero del país como G9 (Galápagos, Manabí, Santo Domingo de los Tsáchilas, Tungurahua, Cotopaxi, Chimborazo, Napo y Orellana, etc.)

\section{Notas}

1 Las ciudades se consideran intermedias no solo con arreglo a tallas demográficas y dimensiones determinadas (coherentes con su contexto geográfico), sino, sobre todo, con relación a las funciones que desarrollan: el papel de mediación en los flujos (bienes, información, innovación, administración, etc.) entre los territorios rurales y urbanos de su área de influencia y los otros centros $\mathrm{o}$ áreas, más o menos alejados. Carmen BELLET SANFELIU \& Josep María LLOP TORNÉ (2004)

\section{Referencias}

Bellet, C., \& Llop, J. M. (2004). Miradas a otros espacios urbanos: las ciudades intermedias. Scripta Nova: revista electrónica de geografía y ciencias sociales, 2004, vol. 8, núm. 165 , p. 1-28.

Camino, M., A., \& Sáinz, J. L. (2012). Desarrollo económico social, cultural y procesos de gobierno de las ciudades intermedias (CIMES). Comparación entre dos ciudades: Valladolid-España y MantaEcuador.

Camino, M., \& Sáinz, J. L. (2013). Hábitat social, digno, sostenible y seguro en Manta, Manabí, Ecuador. Proyecto AECID. 
Universidad Laica Eloy Alfaro de Manabí. Manta.

Instituto Nacional de Estadísticas y Censos

INEC Ecuador (2010) Censos de Población

y Vivienda.

Ministerio de Desarrollo Urbano y Vivienda (2015). Informe Nacional. Conferencia de las Naciones Unidas sobre la Vivienda y el Desarrollo Urbano Sostenible (Hábitat III).

Quito: Ecuador. 